\title{
Sûreté nucléaire : droit de regard et devoir de progrès
}

\author{
Par Michel LAVÉRIE, \\ Directeur de la Sûreté des Installations Nucléaires \\ au Ministère de I'Industrie et du Commerce Extérieur.
}

'in dustrie nucléaire a un intérêt vital à ce que les jugements de l'opinion soient les plus conformes possibles à la réalité des installations. En particulier les progrès de la sûreté doivent être accompagnés des progrès de sa perception par l'ensemble des regards extérieurs.

Anticipation, réalisme, pression de l'opinion, quelles qu'en soient les raisons, un constat s'impose : la France s'est lancée depuis quelques années dans une politique de transparence exigeante. Cette politique se caractérise par la reconnaissance d'un droit de regard sur les choix du nucléaire et notamment sur sa sûreté. Elle implique que la "transparence" ne soit pas vécue comme une contrainte à court terme, mais comme un apport à plus long terme. D'une façon générale, les divers regards extérieurs sur la sûreté nucléaire doivent être reconnus comme bénéfiques Avec des approches différentes, ils évitent la sclérose et les tentations d'autosatisfaction des spécialistes. Ainsi, les doutes, les réflexions, les contre-expertises, les critiques venant des élus, des associations concernées, des organisations syndicales, d'experts, d'organismes internationaux et de bien d'autres partenaires font progresser la sûreté. Les informations qu'ils émettent ou qu'ils relaient sont aussi des facteurs de progrès.

En référence aux événements historiques que nous suivons à l'Est, n'y a-t-il pas un lien entre un "système" et l'accident du 26 avril 1986 sur le réacteur $n^{\circ} 4$ de Tchernobyl? N'assistons-nous pas à l'effondrement d'un mode de pensée qui n'affichait pas les réalités et qui laissait peu de place aux nuances, aux doutes et aux remises en cause qui sont en particulier les qualités de base d'une bonne démarche de sûreté?

e "risque nul" est inaccessible, dans la chaîne des installations concourant à la production d'énergie électronucléaire, comme dans d'autres activités humaines. Certes, mais quel est, à un instant donné, le niveau de risque "acceptable", "aussi bas que raisonnablement possible", «négligeable», selon une série de formulations habituellement employées? "How safe is safe enough?" en est le raccourci intraduisble dans un français concis.

Cette réflexion passe bien entendu par l'évaluation du risque. Les études probabilistes de sûreté réalisées par Electricité de France et par I'Institut de protection de sûreté nucléaire montrent par exemple qu'il y a, en ordre de grandeur, une "chance" sur dix pour que le cœur d'un des 50 réacteurs du parc électronucléaire français soit endommagé, au cours de toute la durée de vie de ce parc.

$\mathrm{Ce}$ chiffre est relativement faible, d'autant que l'accident de Three Mile Island, en 1979, a montré qu'un tel accident, survenant dans un réacteur doté d'une enceinte de confinement efficace, pouvait ne pas avoir de conséquences sanitaires. Mais, en même temps, ce chiffre est suffisamment important pour engendrer un devoir de progrès, d'une part, sur le parc actuel et, d'autre part, sur la prochaine génération de réacteurs.

Sur le parc actuel de réacteurs, comme sur les autres installations du cycle; des gains importants ont déjà été réalisés compte tenu de la conception adoptée. Le gain marginal sera inévitablement de plus en plus difficile. Mais un certain nombre de gisements de progrès existent, notamment par une qualité des réalisations encore plus rigoureuse, par une maintenance améliorée, par une action hiérarchique généralisée visant à promouvoir la rigueur de l'exploitation et en toutes circonstances le caractère inviolable des règles affichées, par une meilleure définition des parades permettant de réduire les conséquences des situations accidentelles, par une "culture de sûreté " accrue de tous les intervenants,..

Pour les installations futures, il convient de se fixer des objectifs de sûreté plus ambitieux : une réduction significative de la production de rejets et de déchets en fonctionnement normal, ainsi que des doses reçues par le personnel; une diminution importante de la probabilité d'accident impliquant notamment des installations plus simples à exploiter, avec des systèmes de sûreté plus passifs; dans I'hypothèse où un accident se produirait cependant, un confinement plus fiable et plus efficace afin de limiter au maximum les rejets dans l'environnement.

La volonté conjointe et persistante des différents partenaires (exploitants, constructeurs, organismes de recherches, autorité de sûreté) est une condition nécessaire de l'aboutissement. Cette ambition dépassera le niveau national et impliquera d'étroites coopérations internationales et notamment européennes.

Dans ce contexte, la "sur-réglementation" ne paraît pas pouvoir être un moteur de ces progrès de sûreté. Au contraire, les excès d'encadrement réglementaire engendreraient un risque de "déresponsabilisation " des exploitants et des constructeurs dont la compétence et le sens des responsabilités demeurent essentiels. L'option fondamentale sur laquelle repose, dans notre organisation française, la relation entre l'exploitant et autorité de sûreté doit être affichée clairement. Aux pouvoirs publics de déterminer des objectifs de sûreté, de plus en plus cohérents, voire unifiés, au plan international. A l'exploitant de définir les modalités techniques pour les atteindre. Aux pouvoirs publics de s'assurer, par des analyses de sûreté, de la bonne adéquation de ces modalités, en regard des objectifs définis. A l'exploitant de mettre correctement en œuvre ces modalités approuvées. Aux pouvoirs publics de vérifier par sondage la qualité de cette mise en œuvre et d'en tirer les conséquences réglementaires.

Cet enchaînement de rôles nécessite un dialogue technique permanent, approfondi et rude. L'efficacité de l'action réglementaire ne doit donc pas résider dans un encadrement technique étroit, mais dans des interactions entre partenaires responsables.

D ans ce contexte, je me réjouis que la Revue Générale Nucléaire ait pris l'initiative d'un numéro spécial consacré à la sûreté nucléaire en France. Ce numéro photographie ainsi l'état d'une réflexion en constante évolution. II y contribue aussi en renvoyant à chacun des acteurs cette juxtaposition de points de vue. 